\title{
Merging Roles in Coordination and in Agent Deliberation.
}

\author{
Guido Boella ${ }^{1}$, Valerio Genovese ${ }^{1}$, Roberto Grenna ${ }^{1}$, and \\ Leendert van der Torre ${ }^{2}$ \\ ${ }^{1}$ Dipartimento di Informatica - Università di Torino - Italy \\ guido@di.unito.it, valerio.click@gmail.com, grenna@di.unito.it \\ ${ }^{2}$ University of Luxembourg, Luxembourg \\ leendert@vandertorre.com
}

\begin{abstract}
In this paper we generalize and merge two models of roles used in multiagent systems which address complementary aspects: enacting roles and communication among roles in an organization or institution. We do this by proposing a metamodel of roles and specializing the metamodel to fit the two existing models. We show how the two approaches can be integrated since they deal with complementary aspects: roles in organizations are used to specify interactions among agents, and, thus, they emphasize the public character of roles. Role enactment focuses instead on how roles are played, and thus it emphasizes the private aspects of roles: how the beliefs and goals of the roles can become the beliefs and goals of their players. The former approach focuses on the dynamics of roles in function of the communication process. The latter approach focuses on the internal dynamics of the agents when they start playing a role or shift the role they are currently playing.
\end{abstract}

\section{Introduction}

In the last years, the usefulness of roles in designing agent organizations has been widely acknowledged. Witness not only the papers appeared at AAMAS, IAT, but also the creation of specialized workshops which have agent organizations (COIN, ROLES, AOSE, NorMAS, etc.) among their topics.

Many different models have been designed. Some of them use roles only in the design phases of a MAS [3], while other ones consider roles as first class entities which exist also during the runtime of the system [4]. Some of them focus on how roles are played by agents [2], other ones on how roles are used in communication among agents in organizations [1].

This heterogeneity of the way roles are defined and used in MAS risks to be a danger for the interoperability of agents in open systems, since each agent entering a MAS can have a radically different notion of role. Thus, the newly entered agents cannot be governed by means of organizations regulating the MAS. Imposing to all agent designers a single notion of role is a strategy that cannot 
have success. Rather, it would be helpful to design both multiagent infrastructures that are able to deal with different notions of roles, and to have agents that are able to adapt to open systems which use different notions of roles in the organizations they set up. This alternative strategy can be costly if it is not possible to have a general model of roles that is compatible, or can be made compatible, with other existing concepts.

In this paper we generalize and merge two models of roles used in MAS, in order to promote the interoperability of systems. The research question is: How to combine the model of role enactment by proposed by [2] with the model of communication among organizational roles of [1]?

We answer these questions by extending to agents a metamodel of roles applied up to now to object oriented systems [5]. The relevant questions, in this case, are: how to introduce beliefs, goals and other mental attitudes in objects, and how to pass from the method invocation paradigm to the message passing paradigm.

Then we specialize the metamodel to model the two existing approaches and we show how they can be integrated in the metamodel since they deal with complementary aspects. We choose to model the proposals of [1] and [2] since they are representative of two main traditions. The first tradition is using roles to model the interaction among agents in organizations, and the second one is about role enactment, i.e., to study how agents have to behave when they play a role.

From one side, organizational models are motivated by the fact that agents playing roles may change, for example a secretary may be replaced by another one if she is ill. Therefore, these models define interaction in terms of roles rather than agents. In [1] roles model the public image that agents build during the interaction with other agents; such image represents the behavior agents are publicly committed to. However, this model leaves unspecified, how given a role, its player will behave. This is a general problem of organizational models which neglect that when, for example, a secretary falls ill, there are usually some problems with ongoing issues (the new secretary does not know precisely the thing to be done, arrangements already made etc.). So having a model of enacting and deacting agents surely leads to some new challenges, which could not be discussed, simulated or formally analyzed without this model.

In contrast, the organizational view focuses on the dynamics of roles in function of the communication process: roles evolve according to the speech acts of the interactants, e.g. the commitment made by a speaker or the commands made by other agents playing roles which are empowered to give orders. In this model roles are modeled as sets of beliefs and goals which are the description of the expected behavior of the agent. Roles are not isolated, but belongs to institutions, where constitutive rules specify how the roles change according to the moves played in the interactions by the agents enacting the roles.

[2] focuses, instead, on how roles are played by an agent, and, thus, on the private aspects of roles. Given a role described in terms of beliefs, goals, and other components, like plans, their model describe how these mental attitudes become 
the beliefs and goals of the agents. In this model roles are fixed descriptions, so they do not have a dynamics like in the model of [1]. Moreover, when roles are considered inside organizations new problems for role enactment emerge: for example, how to coordinate with the other agents knowing what they are expected to do in their role, and how to use the powers which are put at disposal of the player of the role in the organization. The same role definition should lead to different behaviors when the role is played in different organizations.

In contrast, it specifies the internal dynamics of the agents when they start playing (or enacting in their terminology) a role or shift the role they are currently playing (called the activated role). So they model role enacting agents: agents that know which roles they play, the definitions of those roles, and which autonomously adapt their mental states to play the roles.

Despite the apparent differences, the two approaches are compatible since they both attributes beliefs and goals to roles. So we study by means of the metamodel how they can be combined to have a more comprehensive model of roles.

The paper is structured as follows. In Section 2 we describe the requirements on agents and roles in order to build a metamodel; in Section 3 we formally define the metamodel for roles together with its dynamics; in Section 4 we define the basic notions to model agents that play roles; Section 5 deals with the modeling of enacting agents as in [2]; Section 6 introduces and models roles to deal with coordination in organizations; in Section 7 we merge [2] and [1] into the framework introduced in Section 3; Conclusions end the paper.

\section{$2 \quad$ Agents and roles}

Since the aim of this paper is to build a metamodel to promote interoperability, we make minimal assumptions on agents and roles.

The starting point of our proposal is a role metamodel for object orientation. The relation of objects and agents is not clear, and to pass from object to agents we take inspiration from the Jade model [6].

Agents, differently than objects, do not have methods that can be invoked starting from a reference to the object. Rather, they have an identity and they interact via messages. Messages are delivered by the MAS infrastructure, so that agents can be located in different platforms. The messages are modeled via the usual send-receive protocol. We abstract in the metamodel from the details of the communication infrastructure (whether it uses message buffers, etc.).

Agents have beliefs and goals. Goals are modeled as methods which can be executed only by the agent itself when it decides to achieve the goal.

As said above, we propose a very simple model of agents to avoid controversial issues. When we pass to roles, however, controversial issues cannot be avoided.

The requirements to cope with both models of roles we want to integrate are:

- Roles are instances, associated in some way to their players.

- Roles are described (at least) in terms of beliefs and goals. 
- Roles change over time.

- Roles belong to institutions, where the interaction among roles is specified.

- The interaction among roles specifies how the state of roles changes over time.

In [1] roles are used to model interaction, so agents exchange messages according to some protocol passing via their roles. This means that the agent have to act on the roles, e.g., to specify which is the move the role has to play in certain moment. Moreover, roles interact with each other.

[2]'s model specifies how the state of the agent changes in function of the beliefs and goals of the roles it plays. However, it does not consider the possibility that the state of the role change and, thus, it ignores how the agent becomes aware of the changes of beliefs and goals of the role.

To combine the two models we have to specify how the interaction between an agent and its role happens when the agent changes the state of the role or the state of the role is changed by some event. A role could be considered as an object, and its player could invoke a method of the role. However, this scenario is not possible, since the roles are strictly related to the institution they belong to, and we cannot assume that the institution and all the agents playing roles in the institution are located on the same agent platform. So method invocation is not possible unless some sophisticated remote method invocation infrastructure is used. Moreover, the role have to communicate with its player when its beliefs and goals are updated. Given that the agent is not an object, the only possibility is that a role sends a message to its player. As a consequence, we decide to model the interaction between the agent and the role by means of messages too.

Finally, we have to model the interaction among roles. Since all roles of an institution belongs to the same agent platform, they do not necessarily have to communicate via messages. To simplify the interaction, we model communication among roles by means of method invocation.

The fact that roles belong to an institution has another consequence. According to the powerJava model of roles in object oriented programming languages, roles, seen as objects, belong to the same namespace of the institution. This means that each role can access the state of the institution and of the sibling roles. This allows to see roles as a way to specify coordination [7].

In a sense, roles are seen both as objects, from the internal point of view of the institution they belong to, and as agents, from the point of view of their players, with beliefs and goals, but not autonomous. Their behavior is simply to:

- Receive the messages of their players.

- Execute the requests of their player of performing the interaction moves according to the protocol allowed by the institution in that role.

- Send a message to their players when the interaction move performed by the role itself or by some other role results in a change of state of the role. 


\section{A Logical Model for Roles}

In [5] the model is structured in three main levels: universal, individual and dynamic; here we decide not to talk about the universal level an concentrate ourself on agents dynamics. We define the formalism of the framework in a way as much general as possible, this gives us an unconstrained model where special constraints are added later.

\subsection{Individual level}

The individual level includes in this paper some elements of the universal one and the elements of this level are individuals (or instances) of the types defined at the universal level. In the formalization of the model we use objects as basic elements upon which the model is based; we refer to Section 4 for a complete discussion that underlines how the model can be used to grasp roles dynamics in MAS.

Definition 1 A snapshot model is a tuple

$$
\begin{gathered}
<\text { O, R_types, I_contexts, I_players, I_roles, Val, I_contraints } \\
\text { I Roles }_{\text {I_Attributes, I_Operations, I Ittr }}>
\end{gathered}
$$

where:

- $\mathrm{O}$ is a domain of objects, for each object $o$ is possible to refer to its attributes and operations through $\pi_{I_{-} A t t r}(o)$ and $\pi_{I_{-} O p}(o)$, respectively.

- R_types is a set of types of roles.

- I_contexts $\subseteq \mathrm{O}$ is a set of institutions.

- I_players $\subseteq \mathrm{O}$ is a set of actors.

- I_roles $\subset \mathrm{O}$ is a set of roles instances.

- I_Attributes is the set of attributes.

- I_Operations is the set of operations.

- Val is a set of values.

- I_constraints is a set of integrity rules that constraint elements in the snapshot.

We usually refer elements in I_contexts, I_players and I_roles respectively, institutions, actors and roles_instances.

The snapshot model has also a few functions and relations:

- $I_{\text {Roles }}$ is a role assignment function that assigns to each role $R$ a relation on I_context $\times$ I_players $\times$ I_roles.

- $I_{A t t r}$ is an assignment function which it takes as arguments an object $d \in \mathrm{O}$, and an attribute $\mathrm{p} \in \pi_{I_{-} A t t r}(d)$, if $\mathrm{p}$ has a value $v \in$ Val it returns it, $\emptyset$ otherwise.

- I_AS $\subseteq \mathrm{O} \times$ I_Attributes: is an attribute assignment relationship, through which we define what are the attributes assigned to an object in the defined snapshot. 
- I_OS $\subseteq \mathrm{O} \times$ I_Operations: is an operations assignment relationship, through which we define what are the operations assigned to an object in the defined snapshot.

- I_OT $\subseteq \mathrm{O} \times \mathrm{R}$ _types: is an type assignment relationship, through which we define the type of every role instance in the snapshot.

- I_PL $\subseteq$ I_players $\times$ R_types: this relation states, which are the players that can play a certain role types.

- I_RO $\subseteq$ I_roles $\times$ I_contexts: each role is linked with one or (potentially more) context.

Generally, when a role instance $x$ is an individual of the type $D$, we write $x:: D$. If $a \in \pi_{I_{-} A t t r}(x)$ we write $\mathrm{x} . \mathrm{a} \in \mathrm{I}_{-}$Attributes as the attribute instance assigned to object $x$, the same holds for elements in I_Operations.

The role assignment function $I_{\text {Roles }}$ gives us the notion of an actor who plays a role within a specific context: if $i$ is an institution, $a$ an actor, and $o:: R$ a role, $(i, a, o) \in I_{\text {Roles }}(R)$ is to be read as: "the object $o$ represents agent $a$ playing the role $R$ in institution $i$ ". We will often write $R(i, a, o)$ for this statement, and we call $o$ the role instance.

Suppose we have a role instance employee, and that the value of the attribute salary is $1000 €$ usually, instead of writing $I_{A t t r}$ (employee, salary) $=1000$, we write

$$
\text { salary }(\text { employee })=1000
$$

\subsection{The dynamic model}

The dynamic model defines a structure to properly describe how the framework evolves as a consequence of executing an action on a snapshot. In Section 4 and 5 , we describe how this model constraints agents' dynamics.

Definition 2 A dynamic model is a tuple

$<\mathrm{S}, \mathrm{TM}$, Actions, Requirements, D_constraints, $\mathrm{I}_{\text {Actions }}, \mathrm{I}_{\text {Roles }} \pi_{\text {Req }}, I_{\text {Requirements }}>$

where:

$-\mathrm{S}$ is a set of snapshots.

- TM $\subseteq \mathrm{S} \times \mathbb{N}$ : it is a time assignment relationship, such that each snapshot has an associated unique time $t$. For the sake of simplicity we define a discrete time through positive natural numbers.

- Actions is a set of actions.

- Requirements is a set of requirements for playing roles in the dynamic model.

- D_contraints is a set of integrity rules that constraints the dynamic model.

- I Actions maps each action from Actions to a relation on a set of snapshots P. $I_{\text {Actions }}(s, a, t)$ tells us which snapshots are the result of executing action $a$ at time $t$ from a certain snapshop. ${ }^{1}$ This function returns a couple in TM

\footnotetext{
${ }^{1}$ Notice that given an action, we can have several snapshots because we model actions with modal logic in which, from a world it is possible to go to more than one other possible world. This property is often formalized through the accessibility relationship. Thus, each snapshot can be seen as a possible world in modal logic.
} 
that binds the resulting snapshot with time $t+1$. In general, to express that at time $t$ is carried action $a$ we write $a_{t}$.

- About $I_{\text {Roles }_{t}}$ we say that $R_{t}(i, a, o)$ is true if there exists, at a time $t$, the role instance $R(i, a, o)$.

$-\pi_{R e q}(t, R)$ returns a subset of Requirements present at a given time $t$ for the role of type $R$, which are the requirements that must be fulfilled in order to play roles of type $R$.

Intuitively, the snapshots in $\mathrm{S}$ represent the state of a system at a certain time. Looking at $\mathrm{I}_{\text {Actions }}$ is possible to identify the course of actions as an ordered sequence of actions such that:

$$
a_{1} ; b_{2} ; c_{3}
$$

represents a system that evolves due to the execution of $a, b$ and $c$ at consecutive times. We refer to a particular snapshot using the time $t$ as a reference, so that for instance $\pi_{I_{-} A t t r_{t}}$ refers to $\pi_{I_{-} \text {Attr }}$ in the snapshot associated with $t$ in TM.

Actions are described using dynamic modal logic [8], in paricular they are modelled through precondition laws and action laws of the following form:

$$
\begin{gathered}
\square(A \wedge B \wedge C \supset\langle d\rangle \top) \\
\square\left(A^{\prime} \wedge B^{\prime} \wedge C^{\prime} \supset[d] E\right)
\end{gathered}
$$

Where the $\square$ operator express that the quantified formulas hold in all the possible words. Precondition law (1) specifies the conditions $A, B$ and $C$ that make an atomic action $d$ executable in a state. (2) is an action law ${ }^{2}$ which states that if preconditions $A^{\prime}, B^{\prime}$ and $C^{\prime}$ to action $d$ holds, after the execution of $d$ also $E$ holds.

In addition we introduce complex actions which specify complex behaviors by means of procedure definitions, built upon other actions. Formally a complex action has the following form:

$$
\left\langle p_{0}\right\rangle \varphi \subset\left\langle p_{1} ; p_{2} \ldots ; p_{m}\right\rangle \varphi
$$

$p_{0}$ is a procedure name, ";" is the sequencing operator of dynamic logic, and the $p_{i}$ 's, $i \in[1, m]$, are procedure names, atomic actions, or test actions ${ }^{3}$.

Now we show some examples of actions that can be introduced in the dynamic model in order to specialize specialize the model.

\section{Role addition and deletion}

For role addition and deletion actions we use, respectively $R, i \hookrightarrow_{t} \quad a$, and $R, i \hookleftarrow_{t} a$. Then using the notation of dynamic logic introduced above, we write:

$$
\square\left(\operatorname{Req}_{t}(i, a, R) \supset\left\langle R, i \hookrightarrow_{t} a\right\rangle \top\right)
$$

\footnotetext{
${ }^{2}$ Sometimes action laws are called effect rules because E can be considered the effect of the execution of $d$.

${ }^{3}$ Test actions are of the form $\langle\psi ?\rangle \varphi \equiv \psi \wedge \varphi$.
} 
to express that, if actor $a$ fills the requirements at time $t\left(\operatorname{Req}_{t}(i, a, R)\right.$ is True), $a$ can execute the role addition action that let him play role of type $\mathrm{R}$.

The above definition gives us the possibility to model that a role assignment introduces a role instance:

$$
\square\left(\top \supset\left[R, i \hookrightarrow_{t} a\right] \exists x R_{t+1}(i, a, x)\right)
$$

or the fact that if $a$ does not already play the role $R$ within institution $i$, then the role assignment introduces exactly one role instance:

$$
\square\left(\neg \exists x R(i, a, x) \supset\left[R, i \hookrightarrow_{t} a\right] \exists ! x R_{t+1}(i, a, x)\right)
$$

\section{Methods}

There are other actions through which is possible to change the model as well, for instance agents may assign new values to their attributes [5]. Again, the effects of such changes may depend on choices made earlier (e.g. in the case of delegation, changing the attribute value of an object may change the value of that attribute also in some roles he plays).

Here, we will focus on the case in which the attribute's values can be changed by the objects themselves. What we will do is to define methods of objects with which they can change attributes of their own or those of others. Actually, to simplify the model, we define one single primitive action: $\operatorname{set}_{t}\left(o_{1}, o_{2}\right.$, attr, $\left.v\right)$, which means that agent $o_{1}$ sets the value of attr on agent $o_{2}$ to $v$ at time $t$.

Now, we will of course have that:

$$
\square\left(\top \supset\left[\operatorname{set}_{\mathrm{t}}\left(\mathrm{o}_{1}, \mathrm{o}_{2}, \operatorname{attr}^{\mathrm{v}}\right)\right] \operatorname{attr}_{\mathrm{t}+1}\left(\mathrm{o}_{2}\right)=\mathrm{v}\right)
$$

which means that in any state, after the execution of set, if the action of setting this attribute succeeds, then the relevant object will indeed have this value for that attribute.

\section{Operations}

Elements of our framework come with operations that can be executed at the individual level in order to change the model dynamically, the semantics of each operations can be given exploiting the actions defined for the dynamic model. Suppose, for instance, to have an object individual $x$ :: Person with $x$.mail_address attribute, and an operation x.change_mail that changes the value of x.mail_address to its argument. Using the set primitive is possible to define how the model evolves after the execution of x.change_mail operation trough the following axiom:

$$
\left[\mathrm{x} \text {.change_mail } \mathrm{t}_{\mathrm{t}}(\mathrm{s})\right] \varphi \equiv\left[\operatorname{set}_{\mathrm{t}}(\mathrm{x}, \mathrm{x}, \text { mail_adress, } \mathrm{s})\right] \varphi
$$

Where $\mathrm{x}$.change_mail $(\mathrm{s})$ identifies the action carried by $x$ at time $t$ to execute the instance operation x.change_mail; objects can execute only operations that 
are assigned to them by I_OS relation. In Section 5 we define exec of certain operations as complex actions because we have to describe a more complex semantics.

\section{Roles in Multiagent Systems}

Since here we have been talking about objects as cornerstone of the individual level, now in order to switch from objects to agents, it must be underlined that an object of the meta-model does not necessarily overlap with object in OO programming. We used the terms object to refer to individuals, and terms like attribute and operation to talk about state and bheavior of an entity.

In order to be as much general as possible, we define elements of the metamodel with only those features that are essential to talk about roles and leave the possibility to specify the abstract model depending on which account of role we want to grasp. This appraoch gives us the possibility to talk about object and agent using the same framework, and specifing each time which are the charateristics of role's player. In moving form objects to agents we need to state the following:

- Attributes are complex properties of the agent which describe its internal features as well as its mental attitudes (belief, goals, plans etc.).

- Operations are actions that the agent does in the system.

- Agents at individual level are supposed to be autonomous so they cannot be forced to execute an action from an external entity.

- The only way to interact between agents is through message passing.

- The system in which agents interacts is represented by a unique institution.

- Role instances are linked with one and only one system. In order to express this point we add into I_Constraint of every snapshot the following integrity rule:

$$
r \in \mathrm{I} \_ \text {roles } \leftrightarrow \exists ! c \in \mathrm{I}_{\text {_contexts }:}: r, c>\in \mathrm{I} \_\mathrm{RO}
$$

For the sake of generality, we prefer not to specify how agents reason on the basis of their mental attitudes; what we want to model is how mental attitudes evolve as a consequence of playing a role and what are the elements on which the agent have to carry out its resoning process.

It is important to understand that the meta-model is not a framework for agent specification, the elements listed above are the basic features that we think are foundamental to talk about role in MAS, but of course they are not sufficient to utterly model agents.

\section{$5 \quad$ Enact and Deact Roles}

In [2], the problem of formally defining the dynamics of roles, is tackled identifing the actions that can be done in a open system such that agents can enter and leave. In this setting roles have existence outside the agents in the implemented 
system, so "agents are not completely defined by the roles they play"[2]. This view leads to a defnition of roles that sees them as strictly linked with a system (context), instantiable and with their own proper identity.

In [2] four operations to deal with role dynamics are defined: enact and deact, which mean that an agent starts and finishes to occupy (play) a role in a system, and activate and deactivate, which means that an agent starts executing actions (operations) belonging to the role and suspends the execution of the actions.

Although is possible to have an agent with multiple roles enacted simultaneously, only one role can be active at the same time.

Before diving into modeling the four basic operations to deal with roles, we need to match with our framework a few concept defined in [2], following we report a list of elements together with their definition and then how they fit in our meta-model:

- Multiagent system: In [2] roles are taken into account at the implementation level of open $M A S$, they belong to the system which can be entered or left by agents dynamically. In our framewor is possible to view a system as a context to which are linked all roles that can be played by the agents.

- Agent role: A role is a tuple $\langle\sigma, \gamma, \omega\rangle$. Where $\sigma$ are beliefs, $\gamma$ goals and $\omega$ rules representing conditional norms and obligations. This definition specifies a role "in terms of the information that becomes available to agents when they enact the role, the objectives or responabilities that the enacting agent should achieve or satisfy, and normative rules which can for example be used to handle these objectives" [2]. With this view we define, for roles of our framework, a set of complex attributes $\{$ beliefs, goals, plans, rules $\} \in$ I_Attr toghether with the operations that represent actions that an agent can carry out when it activates the roles instance chosing it from the set of roles it is playing.

- Agent type: We consider an agent type "as a set of agent roles with certain constraints and assume that an agent of a certain type decides itself to enact or deact a role". To talk about agent types we use classes instroduced in the framework as a specification of agent instances at the individual level, with this in mind we use the PL relationship to link agent classes to agent roles (role's classes) so that the set of roles that an agent can enact (play), is constrainted by I_PL.

- Role enacting agent: "We assume that role enacting agents have their own mental attitudes consisting of beliefs, goals, plans, and rules that may specify their conditional mental attitudes as well as how to modify their mental attitudes. Therefore, role enacting agents have distinct objectives and rules associated to the active role it is enacting, and sets of distinct objectives and rules adopted from enacted but inactive roles". In our framework we define a role enacting agent as a instance $x$ having a set of attributes $A$ that represent the internal structures used to deliberate.

$$
A=\left\{\text { beliefs }_{\mathrm{a}}, \text { objectives }_{\mathrm{a}}, \text { plans }_{\mathrm{a}}, \text { rules }_{\mathrm{a}} \text {, enacted_roles[], active_role }\right\} \in \pi_{I_{-} \text {Attr }}(x)
$$


The enacted_roles attribute is a role ordered record where each entry with index i corresponds to a triple $\left\langle\sigma_{i}, \gamma_{i}, \omega_{i}\right\rangle$ which represents the set of beliefs, objectives, plans and rules associated to roles instance $i$ enacted by $x$.

As introduced above, the model in [2] identifies four operations to deal with role dynamics, in order to to grasp the foundamental ideas proposed in the cited paper, we redefine the enact, deact, activate and deactivate operations respecting their original meaning. Given a role enacting agent $x$, a role instance $i:: R$ played by $x$ in context $c$ such that,

$$
\begin{gathered}
\text { beliefs }_{\mathrm{r}}, \text { objectives }_{\mathrm{r}}, \text { plans }_{\mathrm{r}}, \text { rules }_{\mathrm{r}} \in \pi_{I_{-} \text {Attr }}(i) \\
\left\{\text { beliefs }_{\mathrm{a}}\right. \text {, objectives } \\
\text { enact, deact, activate, deactivate } \in \pi_{I_{-} O p}(x)
\end{gathered}
$$

Next we report the semantics of each operation exploiting the set primitive:

$$
\begin{aligned}
& \left\langle\text {.enact }_{\mathrm{t}}(\mathrm{i})\right\rangle \varphi \subset\left\langle\mathrm{R}, \mathrm{s} \hookrightarrow \mathrm{x} ; \operatorname{set}_{\mathrm{t}}\left(\mathrm{x}, \mathrm{x}, \text { beliefs }_{\mathrm{a}} \text {, } \text { beliefs }_{\mathrm{a}} \cup \text { beliefs }_{\mathrm{r}}\right) ;\right. \\
& \left.\operatorname{set}_{t}\left(x, x \text {, enacted_roles[i] },<\text { objectives }_{r}, \text { plans }_{r}, \text { rules }_{r}>\right)\right\rangle \varphi \\
& \left\langle x \cdot \operatorname{deact}_{\mathrm{t}}(\mathrm{i})\right\rangle \varphi \subset\left\langle\mathrm{R}, \mathrm{s} \hookleftarrow \mathrm{x} ; \operatorname{set}_{\mathrm{t}}(\mathrm{x}, \mathrm{x}, \text { enacted_roles}[\mathrm{i}], \text { null })\right\rangle \varphi \\
& \left\langle x \text {.activate }{ }_{\mathrm{t}}(\mathrm{i})\right\rangle \varphi \subset\left\langle\operatorname{set}_{\mathrm{t}}(\mathrm{x}, \mathrm{x}, \text { active_role, enacted_roles }[\mathrm{i}])\right\rangle \varphi \\
& \left\langle\text { x.deactivate }_{\mathrm{t}}(\mathrm{i})\right\rangle \varphi \subset\left\langle\operatorname{set}_{\mathrm{t}}(\mathrm{x}, \mathrm{x} \text {, active_role, null })\right\rangle \varphi
\end{aligned}
$$

In order to be coherent it must be respected a logical order in the execution of these operations, as in [2]:

- each operation deact(i) is preceded by a enact(i).

- each operation deactivate(i) is preceded by only one instruction activate(i) that is not preceded by another activate(j).

\section{The public dimension of roles}

In [9] roles are introduced inside institutions to model the interaction among agents. In [1] the model is specifically used to provide a semantics for agent communication languages in terms of public mental attitudes attributed to roles.

The basic ideas of the model are:

- Roles are instances with associated beliefs and goals attributed to them. These mental attitudes are public.

- The public beliefs and goals attributed to roles are changed by speech acts executed either by the role or by other roles. The former case accounts for the addition of preconditions and of the intention to achieve the rational effect of a speech act, the latter one for the case of commands or other speech acts presupposing a hierarchy of authority among roles.

- The agents execute speech acts via their roles. 
This model has been applied to provide a semantics to both FIPA and Social Commitment approaches to agent communication languages [1]. This semantics overcome the problem of the unverifiability of private mental attitudes of agents.

- In order to maintain the model simple enough, we model message passing extending the dynamic model with two actions (methods) send $(x, y, s p)$ and receive $(y, x, s p$. Were send $(x, y, s p)$ should be read as the action carried by $x$ of sending a speech act (sp) to $y$ and similar considerations hold for the receive action.

- A role only listens for the messages sent by the agents playing it:

$\langle$ listen $(\mathrm{r})\rangle \varphi \subset\langle\mathrm{P} ;$ played_by $(\mathrm{r}, \mathrm{x})$ ?; receive $(\mathrm{r}, \mathrm{x}, \mathrm{sp}) ; \mathrm{D}\rangle \varphi$

These rules define a pattern of protocol where $\mathrm{P}$ and $\mathrm{D}$ have to be read as possible other actions that can be executed before and after the receive.

- The reception of a message from the agent has the effect of changing the state of other roles. For example, a command given via a role amounts to the creation of a goal on the receiver if the sender has authority (within the system) over it. ${ }^{4}$

$$
\square \text { authority }_{\text {sys }}(r, \text { request }) \supset\left[\text { receive }\left(r, x, \text { request }\left(r, r^{\prime}, \text { act }\right)\right)\right] \mathbf{G}_{\mathrm{t}}^{r^{\prime}}(\text { act })
$$

- To produce a speech act, the agent has to send a message to the role specifying the illocutive force, the receiver and the content of the speech act:

$\langle$ communicate $(\mathrm{a})\rangle \varphi \subset\langle\mathrm{P} ; \operatorname{send}(\mathrm{x}, \mathrm{r}, \mathrm{sp}) ; \mathrm{D}\rangle \varphi$

\section{$7 \quad$ The combined model}

The two models presented above model complementary aspects of roles: the public character of roles in communication and how agents privately adapt their mental attitudes to the roles they play.

In this section we try to merge the two approaches using the metamodel we presented. On the one hand, the model of [1] is extended from the public side to the private side, by using [2] as a model of role enacting. In this way, the expectations described by the roles resulting from the interaction among agents can become a behavior of agents and they do not remain only a description.

On the other hand, the model of [2] is made more dynamic. In the original model the role is given as a fixed structure. The goals of agent can evolve according to the goal generation rules contained in it, but the beliefs and goals described by the role cannot change. This is unrealistic, since during the activity of the agent enacting its role, it is possible that further information are put at disposal of the role and that new responsibilities are assigned, etc.

${ }^{4}$ request $\left(r, r^{\prime}\right.$, act $)$ is a speech act that has to be read as following: role $r$ asks to $r$ 's player to do act. 
This problem can be overcomed by the merging with the model of [1] and by the addition of a further element, which is anyway necessary in [1]'s model.

First of all, in [2] roles cannot change since they are not related to a more extensive context. Instead, in [1], roles belong to institutions together with other roles. Sibling roles and the institution they belong to are the sources of changes for the role. Second, in [1], the changes of roles are described by the effects of the speech acts which can be performed via roles. These two elements can be added to [2]'s model without apparent contradictions.

The missing element is that both models do not consider the problem of how the player of a role become aware of the changes in the state of the played role as a consequence of the actions of other roles. Furthermore, in [2] a role is given as known by the agent playing the role. This is not a realistic assumption, in particular, when the state of the role changes over time, but also the way an agent comes to know the initial state of the role must be explicitly modeled. Otherwise, all roles instances must be assumed to be publicly known in advance.

In order to merge the two models within the same framework, we need to add (complex) actions which are able to grasp the dynamics introduced in [1] and [2]. Interactions among agents is done through message passing and, in particular, through actions send and receive introduced in section 6 . Next we are going to introduce all the speech-acts and complex actions which are needed to grasp the combined model and then we introduce a running example to clarify their use defining a course of actions in the dynamic model defined in section 3.2.

An agent who wants to play a role within an open system has to ask to the system for a role instance; this process is handled by two speech act: ask_to_play $(R)$ and accept_to_play $(r, A)$, where the first one is sent from the agent to the system in order to ask to play a role of type $\mathrm{R}$, whereas the second is sent from the system to the agent, together with the identifier of the role instance $r$ and a set A of other role instances present in the system, in order to inform the agent with which roles is possible to interact. Next we report the two effect rules associated:

$$
\begin{aligned}
& \square(T \supset \text { [receive(s, x, ask_to_play }(R) \text {; send(s, x, accept_to_play }(r, A)] \\
& \text { played_by }_{\text {sys }}(r, x, s) \\
& \square(T \supset[\operatorname{send}(x, s \text {, ask_to_play }(R) \text {; receive (x, s, accept_to_play }(r, A)] \\
& \text { played_by } \left.{ }_{a g}(r, x, s)\right)
\end{aligned}
$$

Where $s$ is the system, $x$ the agent, and $r$ a role instance of type R. In this section we use $\mathrm{x}, \mathrm{y}, \mathrm{z} \ldots$ to denote agents, $\mathrm{s}$ for the system and $r, r^{\prime}, r^{\prime \prime} \ldots$ for role instances. Notice that played_by sys $_{\mathbf{s}}(r, x, s)$ and played_by ${ }_{a g}(r, x, s)$ refer to two different infrastructures; in Rule 7 is the system that, after having acknowledged the agent request, knows that $x$ is going to play $r$, whereas in Rule 8 is the agent that becomes aware of the play relation between $x$ and $r$. To link the two predicates with the logical model introduced in Section 3 we have that:

$$
\text { played_by }_{\text {sys }}(r, x, s) \wedge \text { played_by }_{\text {ag }}(r, x, s) \rightarrow R(s, x, r)
$$

When we are dealing with a single system we can omit $s$ writing played_by sys $_{\text {s }}(r, x)$ and played_by ${ }_{a g}(r, x)$. 
To enact a role, an agent, provided the identifier of the role instance it wants to enact, has to send a message to the role and to wait till the role replies with the information about the state of the role: its beliefs, goal, plans, etc. When the state is received, the agent can enact the role in the same way described by Rule 3 in Section 5. In order to model such interaction we introduce two complex actions tell_enact, accept_enact and two speech acts accept_enact and inform_enact. Following the specification of the complex actions:

$$
\begin{aligned}
& \langle\text { tell_enact }(\mathrm{x}, \mathrm{r})\rangle \varphi \subset\langle\text { played_byag }(\mathrm{r}, \mathrm{x}) ? ;(\operatorname{send}(\mathrm{a} 1, \mathrm{r} 1 \text {, enact }(\mathrm{x}, \mathrm{r}))\rangle \varphi \\
& \langle\operatorname{accept} \text { enactment }(r, x)\rangle \varphi \subset\left\langle\operatorname{receive}(r, x \text {, enact }(x, r)) \text {; } \operatorname{played} \_b y_{\text {sys }}(r, x) ?\right. \text {; } \\
& \left.\operatorname{send}\left(r, x \text {, inform_enact }\left(<\text { beliefs }_{r} \text {, objectives } r, \text { plans }_{r}, \text { rules }_{r}>\right)\right)\right\rangle \varphi
\end{aligned}
$$

When the agent receives the specification of the role he wishes to enact, it can internalize them as in Rule $3:^{5}$

$$
\begin{array}{r}
\square\left(T \supset\left[\text { receive }\left(x, r, \text { inform_enact }\left(<\text { beliefs }_{r}, \text { objectives }_{r}, \text { plans }_{r}, \text { rules }_{r}>\right)\right)\right]\right. \\
\left.\mathbf{B}^{\times}\left(\text {beliefs }_{r}\right) \wedge \text { x.enacted_roles }[r]=<\text { objectives }_{r}, \text { plans }_{r}, \text { rules }_{r}>\right)
\end{array}
$$

In this combined view is possible that role's specifications change dynamically, in that case it is up to the role to send a message to its player each time its state is updated:

$$
\begin{array}{r}
\langle\text { udpate_state }(r, x)\rangle \varphi \subset\left\langle\text { played_by }_{\text {sys }}(r, x) ? ;\left(\neg \mathbf{G}_{\mathrm{t}}^{r}(\mathbf{q}) \wedge \mathbf{G}_{\mathrm{t}+1}^{r}(\mathrm{q})\right) ? ;\right. \\
\operatorname{send}(r, x, \text { inform_goal }(q))\rangle \varphi
\end{array}
$$

Last but not least, we need to model the deactment of a role respecting the formalization as in Rule 4, therefore we introduce two speech acts deact, ok_deact and a complex action confirm_deact defined as follows:

$$
\begin{array}{r}
\left\langle\operatorname{confirm} \_ \text {deact }(r, x)\right\rangle \varphi \subset\left\langle\text { receive }(r, x, \text { deact }) ; \text { played_by }{ }_{\text {sys }}(r, x) ? ;\right. \\
\operatorname{send}(r, x, \text { ok_deact })\rangle \varphi
\end{array}
$$

After sending the ok_deact, the system will not consider anymore agent $x$ as player of $r$ :

$$
\square\left(\top \supset[\text { confirm_deact }(r, x)] \neg \text { played_by }_{\text {sys }}(r, x)\right.
$$

If it is possible for the agent to deact the role, it will receive an ok_deact from its role:

$$
\square(\top \supset[\text { receive }(x, r \text {, ok_deact })] x \text {.enacted_roles }[r]=\text { null } \wedge \neg \text { played_by }
$$

Fig. 1 depicts two agents which interact through roles in an open system. At time $t$ the system has already agent_B that enacts role $\mathrm{r} 2$ as represented by the black arrow which goes from agent_B to $r 2$. The system evolves as following:

\footnotetext{
${ }^{5}$ Referring to our metamodel introduced in section 3 we have that $\mathbf{B}^{\times}(a)$ is equivalent to state $\mathrm{x}$.beliefs $=\pi_{\mathrm{I}_{-} \text {Attr }}(\mathrm{x}) \cup \mathrm{a}$.
} 


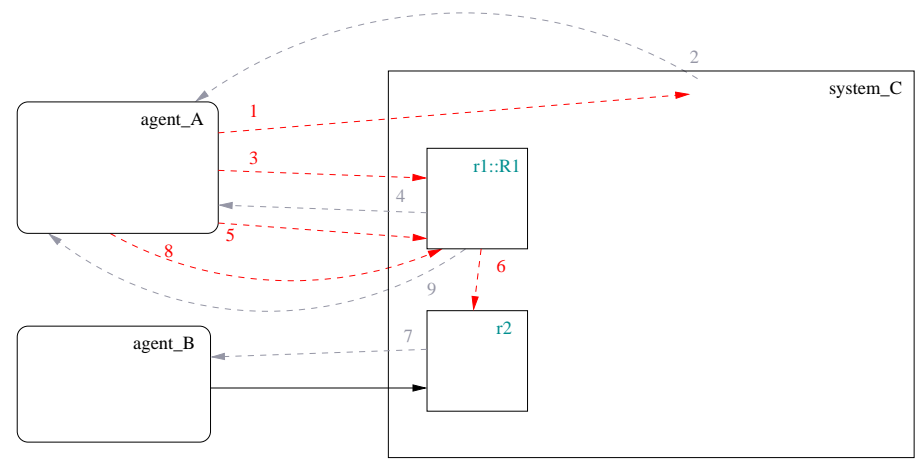

Fig. 1. Roles in MAS

- At time $t+1$ agent_A asks to institution system_C to play a role of type R1:

$$
\text { send }_{t+1}(\text { agent_A, system_C, ask_to_play (R1)) }
$$

- At time $t+2$ system_C replies to agent_A assigning to him the role instance r1:

$$
\operatorname{send}_{\mathrm{t}+2}(\text { system_C, agent_A, accept_to_play }(\mathrm{r} 1,\{\mathrm{r} 2\}))
$$

- At time t+3 agent_A wants to enact (internalize) role r1:

$$
\text { tell_enact }{ }_{\mathrm{t}+3}(\text { agent_A, } r 1)
$$

- At time $t+4$ role $r 1$ receives the speech act from agent_A asking for enactment and accepts it, replying to agent_A with its specifications:

$$
\text { accept_enactment }_{t+4}(\mathrm{r} 1 \text {, agent_A })
$$

- Once that agent_A has enacted the role as in Rule 3 it decides, at time $t+5$, to activate it ${ }^{6}$ and then to ask to the agent playing $\mathrm{r} 2$ to do an action act. In other words:

$$
\operatorname{send}_{t+5}(\text { agent_A, } r 1 \text {, request }(r 1, r 2 \text {, act }))
$$

When $r 1$ receives a send from agent_A asking for an act of $r 2$, first it checks if the sender has the authority in the system to ask such an act, if so r2 acquires the goal to do act:

$$
\operatorname{authority}_{\text {sys }}\left(r^{\prime}, \text { act }\right) \supset\left[\operatorname{receive}\left(r, \operatorname{agent} A \text {, request }\left(r, r^{\prime}, \text { act }\right)\right)\right] \mathbf{G}^{r^{\prime}} \text { (act) }
$$

\footnotetext{
${ }^{6}$ Activating a role means to take into account its specification during the private agent deliberation process, so there is no need to introduce a public action in the dynamic model to represent the activation of a role.
} 
Is important to underline that because role internals are public to other roles in the same system, it is always possible for $\mathrm{r} 1$ to check or modify r2's goals. So, at time $\mathrm{t}+6$ we have:

$$
\text { receive }_{t+6}(r 1 \text {, agent_A, request }(r 1, r 2, \text { act }))
$$

- Now that $\mathrm{r} 2$ has updated its internal state (i.e. its goals) it must inform its player agent_B:

$$
\text { update_state } \mathrm{t}_{\mathrm{t}+7}(\mathrm{r} 2 \text {, agent_B })
$$

Where update_state is modelled as in Rule 12

- At time t+8 agent_A decides to deact the role r1:

$$
\operatorname{send}_{t+8}(\text { agent_A, r1, deact) }
$$

- Finally, at time t+9, r1 confirm the deact:

$$
\text { confirm_deact } t_{t+9}(r 1, \text { agent_A })
$$

\section{Conclusions and Further Works}

In this article we merged two represetative role's models in MAS by introducing a metamodel taken from [5] and adapting it to agents. In particular, we added representations of typical agents' mental attitudes and a framework to deal with message passing. The model has been specialized in order to describe both public and private dimensions of roles $[1,2]$. Finally, we merged the two dimensions defining a group of actions together with their semantics and we modelled a running example to show a possible course of events.

Further works point in two main directions: adapting the proposed metamodel to other roles approaches like [10], and introducing a formal proof theory of roles' actions dynamics and related apects starting from [8].

\section{References}

1. Boella, G., Damiano, R., Hulstijn, J., van der Torre, L.: ACL semantics between social commitments and mental attitudes. In: International Workshops on Agent Communication, AC 2005 and AC 2006. Volume 3859 of LNAI. Springer, Berlin (2006) 30-44

2. Dastani, M., van Riemsdijk, B., Hulstijn, J., Dignum, F., Meyer, J.J.: Enacting and deacting roles in agent programming. In: Procs. of AOSE'04, New York (2004) 189-204

3. Zambonelli, F., Jennings, N., Wooldridge, M.: Developing multiagent systems: The Gaia methodology. IEEE Transactions of Software Engineering and Methodology 12(3) (2003) 317-370

4. Colman, A., Han, J.: Roles, players and adaptable organizations. Applied Ontology (2007) 
5. Valerio Genovese: Towards a general framework for modelling roles. In Guido Boella, Leon van der Torre, Harko Verhagen, eds.: Normative Multi-agent Systems. Number 07122 in Dagstuhl Seminar Proceedings, Internationales Begegnungs- und Forschungszentrum fuer Informatik (IBFI), Schloss Dagstuhl, Germany (2007)

6. Bellifemine, F., Poggi, A., Rimassa, G.: Developing multi-agent systems with a FIPA-compliant agent framework. Software - Practice And Experience 31(2) (2001) 103-128

7. Baldoni, M., Boella, G., van der Torre, L.: Roles as a coordination construct: Introducing powerJava. Electronic Notes in Theoretical Computer Science (ENTCS) Procs. of the First International Workshop on Methods and Tools for Coordinating Concurrent, Distributed and Mobile Systems (MTCoord 2005) 150 (2006) 9-29

8. M. Baldoni, C. Baroglio, A. Martelli, V. Patti: Reasoning about interaction protocols for customizing web service selection and composition. Journal of LOgic and Algebraic Programming, special issue on Web Services and Formal Methods,70(1):53-73 (2007)

9. Boella, G., van der Torre, L.: The ontological properties of social roles in multiagent systems: Definitional dependence, powers and roles playing roles. Artificial Intelligence and Law Journal (AILaw) (2007)

10. Omicini, A., Ricci, A., Viroli, M.: An algebraic approach for modelling organisation, roles and contexts in MAS. Applicable Algebra in Engineering, Communication and Computing 16 (2005) 151-178 